\title{
Air pollution and survival after MI
}

Researchers from the UK have demonstrated an association between exposure to fine-particle air pollution and all-cause mortality among survivors of myocardial infarction (MI). This new study builds on the results of earlier research that has highlighted the detrimental effects of air pollution among the general population.

Data from 154,204 patients in the Myocardial Ischaemia National Audit Project database for England and Wales were analyzed. Patients selected for inclusion in the study had been admitted to hospital between January 2004 and March 2007 with a diagnosis of ST-segment elevation or non-ST-segment elevation MI. During follow-up (mean 3.7 years), 26\% of these individuals died. Exposure was calculated using the postcode of the address where the patient resided at the time of hospital admission. A significant link was found between exposure to particles $<2.5 \mu \mathrm{m}$ in diameter $\left(\mathrm{PM}_{2.5}\right)$ and mortality. However, no such association was shown for larger particles $\left(\mathrm{PM}_{10}\right)$, or for oxides of nitrogen. Although patients who lived in areas of socioeconomic deprivation had the highest level of exposure to air pollution, this relationship did not fully account for the association between low socioeconomic status and mortality.

Inhalation of particulate matter is known to lead to various pathological changes associated with progression of atherosclerosis and inflammation. However, whether a previous ischaemic event increases susceptibility to the effects of air pollution is not known. Cathryn Tonne and Paul Wilkinson who conducted the study speculate that "if [the growing number of] MI survivors are found to be more susceptible, this could have important public health implications". Large studies in the general population are needed to address this question.

\section{Alexandra King}

Original article Tonne, C. \& Wilkinson, P. Long-term exposure to air pollution is associated with survival following acute coronary syndrome. Eur. Heart J. doi:10.1093/eurheartj/ehs480 